\title{
NONLINEAR DYNAMIC RESEARCH OF THE SPACECRAFT ROBUST FAULT TOLERANT CONTROL SYSTEMS *
}

\author{
Ye.I. Somov ${ }^{*, \dagger}$, A.G. Kozlov ${ }^{\ddagger}$, V.A. Rayevsky ${ }^{\ddagger}$, \\ G.P. Anshakov ${ }^{\S}$, Yu.G. Antonov ${ }^{\S}$ \\ * Research Institute of Mechanical Systems Reliability \\ (NII PNMS), Samara State Technical University \\ 244 Molodogvargeyeskaya Str. Samara 443100 Russia \\ somov@icc.ru_pnms@sstu.edu.ru \\ $\dagger$ Stability and Nonlinear Dynamics Research Center, \\ Mechanical Engineering Research Institute (IMash), \\ the Russian Academy of Sciences (RAS) \\ 5 Dm. Ul'yanov Str. Moscow 117333 Russia \\ $\ddagger$ Research \& Production Association "Applied Mechanics" \\ (NPO PM), the Russian Space Agency (RKA) \\ 52 Lenin Str. Krasnoyarsk-26 660026 Russia \\ $\S$ State Research \& Production Rocket-Space Center \\ "TsSKB-Progress", the Russian Space Agency (RKA) \\ 18 Pskovskaya Str. Samara 443009 Russia
}

\begin{abstract}
New results on methods and software for nonlinear dynamic research and design of robust gyromoment fault tolerant control systems by the information spacecraft (remote sensing, communication, navigation and geodesic) developed by the leading Russian State Space Research and Production Associations (RPAs) the TsSKB-Progress and the NPO PM, are presented. Copyright ${ }^{\circ} 2002$ IFAC
\end{abstract}

Keywords: spacecraft, attitude control system, fault tolerance, nonlinear dynamics

\section{INTRODUCTION}

Over the last two decades, the basic research on fault diagnosis, fault detection and isolation (FDI) at general control systems have received much attentions. The model-based approach is now recognized as an important and efficient method (Frank et al., 2000), the trends in extending that methodology to nonlinear control systems today are practically realized at aerospace engineering by most modern motto in Computer Science (Kurakin, 1999; Somov, 2001b):

from Artificial Intelligence to Natural Tricks.

* This work was supported by the RKA and Russian Foundation for Basic Research (RFBR), grant 00-01-00293.
During the recent 30 years authors have been accumulated a substantial experience (Natural Tricks) in designing the spacecraft (SC) attitude control systems (ACSs) with high fault-tolerance, survivability and autonomy at the expense of functional redundancy. The dynamic requirements to the ACS for the communication, navigation and geodesic SCs are:

- continuous precision 3-axis orientation of the SC body by only minimum number of measurements under possible ACS on-board equipment failures, and also in executing a $\mathrm{SC}$ orbit correction;

- possibility of the SC body re-orientation for its orbit correction, as well autonomous control of the solar array panels (SAPs) 
and each high-gain receiving-transmitting antenna (RTA) with respect to the SC body;

- robustness to variations of the SC inertial and rigidity characteristics under minimum mass, size and power expenditures.

For the remote sensing SC these requirements are expressed by rapid angular manoeuvering and a spatial compensating motion with a variable vector of angular rate, there are the need:

- to orient the telescope line-of-sight to a predetermined part of the Earth surface with the scan in designated direction;

- to compensate an image motion at the onboard optical telescope focal plane.

Increased requirements to such information satellites (lifetime up to 10 years, exactness of spatial rotation manoeuvers with the effective damping of the SC flexible construction oscillations, fault tolerance, reliability as well as to reasonable mass, size and energy characteristics) have motivated intensive development the moment gyrocomplex (MGC) based on excessive number of reaction wheels (RWs), gyrowhells (GWs) and gyrodines (GDs) — single-gimbal control moment gyros. Mathematical aspects of the spacecraft nonlinear gyromoment attitude control were represented in a number of research works (Junkins and Turner, 1986). The recent results (Schaub et al., 1998) are based on methods of Lyapunov functions, whereas the exact feedback linearization (EFL) technique also was applied to this problem. This paper suggests original results on nonlinear methods and software for modeling, dynamic research, design and simulation of the SC gyromoment robust fault tolerant attitude control systems.

\section{STATEMENT OF GENERAL PROBLEM}

Let be given the nonlinear generalized controlled object $\mathcal{O}$ for a time $t \in \mathrm{T}_{t_{0}} \equiv\left[t_{0}, \infty\right)$

$$
\begin{aligned}
D^{+} \mathrm{x}(t)= & \mathcal{F}\left(\mathrm{x}(t), \mathrm{u}, \mathrm{p}(t, \mathrm{x}), \gamma_{\nu}^{f}(t)\right), \mathrm{x}\left(t_{0}\right)=\mathrm{x}_{0} \\
& \mathrm{y}(t)=\psi^{o}\left(\mathrm{x}(t), \gamma_{\nu}^{f}(t)\right) \\
& \mathrm{z}^{o}(t)=\phi^{o}(\mathrm{x}(t), \mathrm{y}(t), \mathrm{p}(t, \mathrm{x}))
\end{aligned}
$$

where $\mathrm{x}(t) \in \mathcal{H} \subset \mathbb{R}^{n_{\nu}} ; \mathrm{x}_{0} \in \mathcal{H}_{0} \subseteq \mathcal{H} ; \mathrm{y}(t) \in$ $\mathbb{R}^{r_{\nu}^{s}}$ is an output vector for measurement and diagnosis of object's state, and $\mathrm{z}^{o}(t) \in \mathbb{R}^{r_{\nu}^{f}}$ is a vector for description of its failure conditions; $\mathrm{u}=\left\{u_{j}\right\} \in \mathrm{U} \subset \mathbb{R}^{r_{\nu}^{c}}$ is a control vector, and $\mathrm{p}(t, \mathrm{x}) \in \mathcal{P}$ is the vector-function of disturbances in class $\mathcal{P} ; D^{+}$is symbol of a right derivative with respect to time, and $\gamma_{\nu}^{f}(t) \in \mathcal{B}^{m}$ under $\mathcal{B}^{m} \equiv$ $\mathcal{B} \times \mathcal{B} \cdots \times \mathcal{B}, \mathcal{B}=\{0,1\}$ is vector of logic variables, which are outputs of a "fault's" asynchronous logic automaton (ALA) $\mathcal{A}^{f}$ for its time $\nu \in \mathbb{N}_{0} \equiv$ $[0,1,2, \ldots)$,

$$
\gamma_{\nu}^{f}=\delta^{f}\left(\kappa_{\nu}^{f}, l_{\nu}^{f}\right) ; \kappa_{\nu+1}^{f}=\lambda^{f}\left(\kappa_{\nu}^{f}, \mathrm{l}_{\nu}^{f}\right), \kappa_{0}^{f}=\kappa^{f}(0),
$$

with memory, where logic vectors of object's state $\kappa_{\nu}^{f}=\kappa^{f}(\nu)$ and input $\mathrm{l}_{\nu}^{f}=\mathrm{l}^{f}(\nu)=\mathrm{g}^{f}\left(\mathrm{z}^{o}\left(t_{\nu}^{f}\right)\right)$, which are used for representing fault occurrences and damage development depending on the automaton time $\nu$, bound up with the continuous time as $t=t_{\nu}^{f}+\left(\tau^{f}-t_{\nu}^{f}\right) ; \quad \tau^{f} \in \tau_{\nu}^{f} \equiv\left[t_{\nu}^{f}, t_{\nu+1}^{f}\right)$, $\nu \in \mathbb{N}_{0}$. Moreover, $\mathrm{l}_{\nu}^{f}(t)=$ const $\forall t \in \tau_{\nu}^{f}$ and change of the logic vector $\gamma_{\nu}^{f}$ in general case leads to variation of dimensions for vectors $\mathrm{x}(t)$ and $\mathrm{y}(t)$ under mappings in time moments $t=t_{\nu}^{f}$ :

$$
\mathrm{x}\left(t_{\nu+}^{f}\right)=\mathcal{P}_{\nu}^{x}\left(\mathrm{x}\left(t_{\nu-}^{f}\right)\right) ; \quad \mathrm{y}\left(t_{\nu+}^{f}\right)=\mathcal{P}_{\nu}^{y}\left(\mathrm{y}\left(t_{\nu-}^{f}\right)\right) .
$$

Let $\mathrm{T}_{u}, \mathrm{~T}_{q} \leq \mathrm{T}_{u}$ and $\mathrm{T}_{r} \geq \mathrm{T}_{u}$ are fixed sampling periods of control, state measurement and the control reconfiguration, moreover, multiplicity conditions must be satisfied for these periods, and

$$
\begin{gathered}
x_{k}=x\left(t_{k}\right) ; t_{k}=k \mathrm{~T}_{u}, t_{s}=s \mathrm{~T}_{q}, t_{\mu}=\mu \mathrm{T}_{r} ; \\
x_{k}^{\mathrm{f}}=\mathcal{F}_{\mathrm{T}_{u}}\left(x_{s}\right) ; \quad x_{\mu}^{\mathrm{f}}=\mathcal{F}_{\mathrm{T}_{r}}\left(x_{k}\right),
\end{gathered}
$$

where $x_{k}^{\mathrm{f}}$ is the value of the variable $x_{s}$ measured with the sampling period $\mathrm{T}_{q}$, which is filtered out at the time $t=t_{k} ; \mathcal{F}_{\mathrm{T}_{y}}(\cdot)$ is the digital filtering operator with the sampling period $\mathrm{T}_{y}, y=u, r$.

Let be also given subsystem of discrete measurement of the object state and digital filtering:

- for diagnostics of the object $\mathcal{O}$

$$
\mathrm{y}_{s}^{d}=\psi^{d}\left(\mathrm{y}_{s}\right) ; \mathrm{z}_{k}^{d \mathrm{f}}=\mathcal{F}_{\mathrm{T}_{u}}\left(\mathrm{y}_{s}^{d}\right), k, s \in \mathbb{N}_{0} ;
$$

- for forming the control and its reconfiguration

$$
\begin{aligned}
& \mathrm{y}_{s}^{u}=\psi^{u}\left(\mathrm{y}_{s}\right) ; \quad \mathrm{y}_{k}^{\mathrm{f}}=\mathcal{F}_{\mathrm{T}_{u}}\left(\mathrm{y}_{s}^{u}\right) ; \\
& \mathrm{z}_{\mu}^{\mathrm{f}}=\mathcal{F}_{\mathrm{T}_{r}}\left(\mathrm{z}_{k}^{d \mathrm{f}}\right), \quad \mu, k, s \in \mathbb{N}_{0} .
\end{aligned}
$$

Principal problems are contained in synthesis of:

- the synchronous logic automaton (SLA) $\mathcal{A}^{d}$ with memory for the structural state diagnosis

$$
\gamma_{k}^{d}=\delta^{d}\left(\kappa_{k}^{d}, \mathrm{l}_{k}^{d}\right) ; \kappa_{k+1}^{d}=\lambda^{d}\left(\kappa_{k}^{d}, 1_{k}^{d}\right), \kappa_{0}^{d}=\kappa^{d}\left(t_{0}\right),
$$

with logic vectors of state $\kappa_{k}^{d}$, input $\mathrm{l}_{k}^{d}=\mathrm{g}^{d}\left(\mathrm{z}_{k}^{d \mathrm{f}}\right)$ and output $\gamma_{k}^{d}$;

- the SLA $\mathcal{A}^{r}$, also with memory, for description of damage's block-keeping and reconfiguration

$\gamma_{\mu}^{r}=\delta^{r}\left(\kappa_{\mu}^{r}, \mathrm{l}_{\mu}^{r}\right) ; \kappa_{\mu+1}^{r}=\lambda^{r}\left(\kappa_{\mu}^{r}, \mathrm{l}_{\mu}^{r}\right), \kappa_{0}^{r}=\kappa^{r}\left(t_{0}\right)$,

with logic vectors of state $\kappa_{\mu}^{r}$, input $\mathrm{l}_{\mu}^{r}=$ $\mathrm{g}^{r}\left(\mathrm{z}_{\mu}^{\mathrm{f}}, \gamma_{\mu}^{d \mathrm{f}}\right)$, where $\gamma_{\mu}^{d \mathrm{f}}=\mathcal{F}_{\mathrm{T}_{r}}\left(\gamma_{k}^{d}\right)$, and output $\gamma_{\mu}^{r}$;

- the nonlinear control law (NCL) with its reconfigurations due to the SLA $\mathcal{A}^{r}$ routine

$$
\begin{aligned}
& \mathrm{u}_{k}=\mathcal{U}\left(\hat{\mathrm{x}}_{e k}, \mathrm{y}_{e k}^{\mathrm{f}}, \mathrm{y}_{o k}, \gamma_{\mu}^{r}\right) ; \\
& \hat{\mathrm{x}}_{e k+1}=\hat{\mathcal{F}}_{e}\left(\hat{\mathrm{x}}_{e k}, \mathrm{y}_{e k}^{\mathrm{f}}, \mathrm{y}_{k}^{o}, \mathrm{u}_{k}, \gamma_{k}^{d}, \gamma_{\mu}^{r}\right), \\
& \hat{\mathrm{x}}_{e 0}=\hat{\mathrm{x}}_{e}\left(t_{0}\right) ; \quad k, \mu \in \mathbb{N}_{0},
\end{aligned}
$$

where $\mathrm{y}_{e k}^{\mathrm{f}}=\mathcal{F}_{\mathrm{T}_{u}}\left(\psi_{e}^{u}\left(\mathrm{y}_{e s}\right)\right)$; $\mathrm{y}_{e s}=\psi_{e}^{o}\left(\mathrm{x}_{e s}, \gamma_{k}^{d}\right)$, and $\mathrm{x}_{e s}=\mathrm{x}_{e}\left(t_{s}\right) \in \mathbb{R}^{n_{\mu}^{e}}$ is the state vector of a simplified discrete object's model

$$
\mathrm{x}_{e s+1}=\mathcal{F}_{e}\left(\mathrm{x}_{e s}, \mathrm{u}_{k}, \gamma_{k}^{d}, \gamma_{\mu}^{r}\right), \mathrm{x}_{e 0}=\mathrm{x}_{e}\left(t_{0}\right),
$$

and $\hat{\mathrm{x}}_{e k}=\hat{\mathrm{x}}_{e}\left(t_{k}\right) \in \mathbb{R}^{n_{\mu}^{e}}$ is its estimation; $n_{\mu}^{e} \leq n=$ $\max \left\{n_{\nu}\right\}$, and $\mathrm{y}_{k}^{o}$ is a programmed vector.

Feedback loops (4)-(9) are intended for faulttolerant control of the object (1)-(3). 


\section{SYNTHESIS OF FEEDBACK CONTROL}

Applied general approach to synthesis of nonlinear control systems (NCS) with a partial measurement of their state is presented when their structural state is known. Moreover, the method of vector Lyapunov functions (VLF), which has a strong mathematical basis for analysis of stability and other dynamical properties of various nonlinear interconnected systems with the discontinuous right-hand side, is used in cooperation with the EFL technique (Isidori, 1989).

Let there be given a nonlinear controlled object $D^{+} \mathrm{x}(t)=\mathcal{F}(\mathrm{x}(t), \mathrm{u}, \mathrm{p}(t, \mathrm{x})) ; \mathrm{x}\left(t_{0}\right)=\mathrm{x}_{0} ; t \in \mathrm{T}_{t_{0}}$, where $\mathrm{x}(t) \in \mathcal{H} \subset \mathbb{R}^{n}$ is a state vector with an initial condition $\mathrm{x}_{0} \in \mathcal{H}_{0} \subseteq \mathcal{H} ; \mathrm{u}=\left\{u_{j}\right\} \in \mathrm{U} \subset \mathbb{R}^{r}$ is a control vector, and $\mathrm{p}(t, \mathrm{x}) \in \mathcal{P}$ is the vector-function of disturbances. Let some vector norms $\rho(\mathrm{x}) \in \overline{\mathbb{R}}_{+}^{l}$ and $\rho^{0}\left(\mathrm{x}_{0}\right) \in \overline{\mathbb{R}}_{+}^{l_{0}}$ also be given. For any NCL $\mathrm{u}=\mathcal{U}(\mathrm{x})$ the closed-loop system has the form

$$
D^{+} \mathrm{x}(t)=\mathcal{X}(t, \mathrm{x}) ; \mathrm{x}\left(t_{0}\right)=\mathrm{x}_{0},
$$

where $\mathcal{X}(t, \mathrm{x})=\mathcal{F}(\mathrm{x}, \mathcal{U}(\mathrm{x}), \mathrm{p}(\cdot)), \mathcal{X}: \mathrm{T}_{t_{0}} \times \mathcal{H} \rightarrow \mathcal{H}$ is a discontinuous operator. Assuming the existence and the non-local continuability of the rightsided solution $x(t) \equiv \mathrm{x}\left(t_{0}, \mathrm{x}_{0} ; t\right)$ of the system (10) for its extended definition in the aspect of physics, the most important dynamic property is obtained, that is $\rho \rho^{0}$-exponential invariance of the solution $x(t)=0$ under the desired $\gamma \in \overline{\mathbb{R}}_{+}^{l}$ :

$\left(\exists \alpha \in \mathbb{R}_{+}\right)\left(\exists \mathcal{B} \in \overline{\mathrm{B}}_{+}^{l \times l_{0}}\right)\left(\exists \delta \in \mathbb{R}_{+}^{l_{0}}\right)\left(\forall \rho^{0}\left(\mathrm{x}_{0}\right)<\delta\right)$ $\rho(x(t)) \leq \gamma+\mathcal{B} \rho^{0}\left(\mathrm{x}_{0}\right) \exp \left(-\alpha\left(t-t_{0}\right)\right) \quad \forall t \in \mathrm{T}_{t_{0}}$. For the VLF $v: \mathcal{H} \rightarrow \overline{\mathbb{R}}_{+}^{k}$ with the components $v^{s}(\mathrm{x}) \geq 0, v^{s}(0)=0, s=1: k$ and the norm $\|v(\mathrm{x})\|=\max \left\{v^{s}(\mathrm{x}), s=1: k\right\}$, defined are the scalar function $\bar{v}(\mathrm{x})=\max \left\{v^{s}(\mathrm{x}), s=1: l_{k}, 1 \leq l_{k} \leq k\right\}$ and a lower right derivative with respect to (10):

$$
\underline{v}^{\prime}(\mathrm{x})=\lim _{\delta \overline{t \rightarrow 0}+}\{(v(\mathrm{x}+\delta t \mathcal{X}(t, \mathrm{x}))-v(\mathrm{x})) / \delta t\} .
$$

Theorem. Let there exist the VLF $v$, so that:

1) $\left(\exists \mathrm{a} \in \mathbb{R}_{+}^{l}\right)(\forall \mathrm{x} \in \mathcal{H}) \quad \rho(\mathrm{x}) \leq \mathrm{a} \cdot \bar{v}(\mathrm{x})$;

2) $\left(\exists \mathrm{b} \in \mathbb{R}_{+}^{l_{0}}\right)\left(\forall \mathrm{x}_{0} \in \mathcal{H}_{0}\right)\left\|v\left(\mathrm{x}_{0}\right)\right\| \leq\left\langle\mathrm{b}, \rho^{0}\left(\mathrm{x}_{0}\right)\right\rangle$;

3) $\exists \gamma_{c} \in \overline{\mathbb{R}}_{+}^{k}$ and a function $\varphi_{\gamma}(\cdot)$ exists so that $\gamma_{c} \leq \varphi_{\gamma}(\mathrm{a}, \gamma)$

4) $\forall(t, \mathrm{x}) \in\left(\mathrm{T}_{t_{0}} \times \mathcal{H}\right)$ the conditions are satisfied:

a) $\underline{v}_{\gamma}^{\prime}(\mathrm{x}) \leq \dot{\mathrm{f}}_{c}\left(t, v_{\gamma}(\mathrm{x})\right) \equiv \mathrm{P} v_{\gamma}(\mathrm{x})+\tilde{\mathrm{f}}_{c}\left(t, v_{\gamma}(\mathrm{x})\right)$;

b) Hurwitz condition for the positive matrix $\mathrm{P}$;

c) Wažewski condition on quasi-monotonicity for the function $\tilde{\mathrm{f}}_{c}(t, \mathrm{y})$;

d) Carateodory condition for the function $\tilde{\mathrm{f}}_{c}(t, \mathrm{y})$, bounded in each domain $\Omega_{c}^{r}=\left(\mathrm{T}_{t_{0}} \times \mathcal{S}_{c}^{r}\right)$, where $r>0$ and $\mathcal{S}_{c}^{r}=\left\{\mathrm{y} \in \mathbb{R}^{k}:\|\mathrm{y}\|_{\mathrm{E}}<r\right\}$;

e) $\left(\tilde{\mathrm{f}}_{c}(t, \mathrm{y}) /\|\mathrm{y}\|\right) \stackrel{t \in \mathrm{T}_{t_{0}}}{\Longrightarrow} 0$ for $\mathrm{y} \rightarrow 0$ uniformly with respect to time $t \in \mathrm{T}_{t_{0}}$, where $v_{\gamma}=v-\gamma_{c}$. Then solution $x(t)=0$ of the system (10) is $\rho \rho^{0}$-exponential invariant and the matrix $\mathcal{B}$ has the form $\mathcal{B}=c \cdot \mathrm{ab}^{\mathrm{t}}$ with $c \in \mathbb{R}_{+}$.

Proof see for example in Somov et al. (1999a): the basis of inequality for vector norm $\rho(x(t))$ is attained by the comparison principle, using the maximum right-sided solution $\bar{x}_{c}(t) \equiv \overline{\mathrm{x}}_{c}\left(t_{0}, \mathrm{x}_{c 0} ; t\right)$ of a comparison system.

There is such an important problem: by what approach is it possible to create constructive techniques for constructing the VLF $v(\mathrm{x})$ and $s i$ multaneous synthesis of a nonlinear control law $\mathrm{u}=\mathcal{U}(\mathrm{x})$ for the close-loop system (10) with given vector norms $\rho(\mathrm{x})$ and $\rho^{0}\left(\mathrm{x}_{0}\right)$ ? Recently, a pithy technique on constructing VLF at such synthesis has been elaborated. This method is based on a nonlinear transformation of the NCS model and solving the problem in two stages.

In stage 1 , the right side $\mathcal{F}(\cdot)$ in (10) is transformed as $\mathcal{F}(\cdot)=\mathrm{f}(\mathrm{x})+\mathrm{G}(\mathrm{x}) \mathrm{u}+\tilde{\mathcal{F}}(t, \mathrm{x}(t), \tilde{\mathrm{u}})$, some principal variables in a state vector $\mathrm{x} \in \tilde{\mathcal{H}} \subset \mathbb{R}^{\tilde{n}} \subseteq$ $\mathbb{R}^{n}$ with $\tilde{n} \leq n, \mathrm{x}_{0} \in \tilde{\mathcal{H}}_{0} \subseteq \tilde{\mathcal{H}}$ are selected and a simplified nonlinear model of the object (10) is presented in the form of an affine quite smooth nonlinear control system

$\dot{\mathrm{x}}=\mathrm{F}(\mathrm{x}, \mathrm{u}) \equiv \mathrm{f}(\mathrm{x})+\mathrm{G}(\mathrm{x}) \mathrm{u} \equiv \mathrm{f}(\mathrm{x})+\sum \mathrm{g}_{j}(\mathrm{x}) u_{j}$,

which is structurally synthesized by the EFL technique. In this aspect, based on the structural analysis of given vector norms $\rho(\mathrm{x})$ and $\rho^{0}(\mathrm{x})$, and also vector-functions $\mathrm{f}(\mathrm{x})$ and $\mathrm{g}_{j}(\mathrm{x})$, the output vector-function $\mathrm{h}(\mathrm{x})=\left\{\mathrm{h}_{i}(\mathrm{x})\right\}$ is carefully selected. Furthermore, the nonlinear invertible (one-to-one) coordinate transformation $\mathrm{z}=\Phi(\mathrm{x})$ $\forall \mathrm{x} \in \mathcal{S}_{\mathrm{h}} \subseteq \tilde{\mathcal{H}}$ with $\Phi(0)=0$ is analytically obtained with simultaneous constructing the VLF.

Finally, bilateral component-wise inequalities for the vectors $\mathrm{x}, \mathrm{z}, v(\mathrm{x}), \rho(\mathrm{x}), \rho^{0}\left(\mathrm{x}_{0}\right)$ are derived, it is most desirable to obtain the explicit form for the nonlinear transformation $\mathrm{x}=\Psi(\mathrm{z})$, inverse with respect to $\mathrm{z}=\Phi(\mathrm{x})$, and the VLF aggregation procedure is carried out with analysis of proximity for a singular directions in the Jacobian $[\partial \mathrm{F}(\mathrm{x}, \mathcal{U}(\mathrm{x})) / \partial \mathrm{x}]$.

In stage 2 , the problem of the NCL synthesis for the complete model of the NCS (10), taking rejected coordinates and nonlinearities, and also the restriction on control, into account is solved by the VLF-method.

If a forming control is digital and a measurement of coordinate's state is discrete and incomplete, then a simplified nonlinear discrete object's model is obtained by Taylor-Lie series (Kazantzis and Kravaris, 1997), a nonlinear discrete observer in the NCL is formed and its parametric synthesis is carried out with a simultaneously (Somov, 1997) construct a discrete sub-vector VLF. 


\section{SYNTHESIS OF LOGIC AUTOMATA}

For the FDI a three-level logic-digital system is generally applied on-board Russian spacecraft for remote sensing, communication and navigation:

- on the lower level - the integral local SLAs $\mathcal{A}_{d}^{d}$ with memory for automatic monitoring of the relevant device status by measurement of available physical variables (currents, movements, rates, etc.)

- on the middle level - the local loop SLAs $\mathcal{A}_{c}^{d}$ with memory for automatic monitoring of the control loop status (the roll, yaw and pitch channels, the SAP control loop etc.);

- on the higher "system" level — a SLA $\mathcal{A}^{d}$, also with memory, for the global functional diagnostics of the main control loop by comparison of outputs for normal and emergency models of the ACS operation.

For high fail-safe operation of the ACS, maximum employment of functional redundancy has been provided by using the SLA to apply all the reverse complete sets of devices or their electric circuits.

At synthesis of the diagnosis SLAs $\mathcal{A}_{d}^{d}, \mathcal{A}_{c}^{d}$ and $\mathcal{A}^{d}$ (6) and also of a damage block-keeping \& reconfiguration SLA $\mathcal{A}^{r}(7)$, the Natural Tricks are used. They are based on both well-known physical invariant relations (for example, general momentum invariant for the "SC+MGC"-system) and engineering inventiveness, presented at a perfect logic-uncontraditory form.

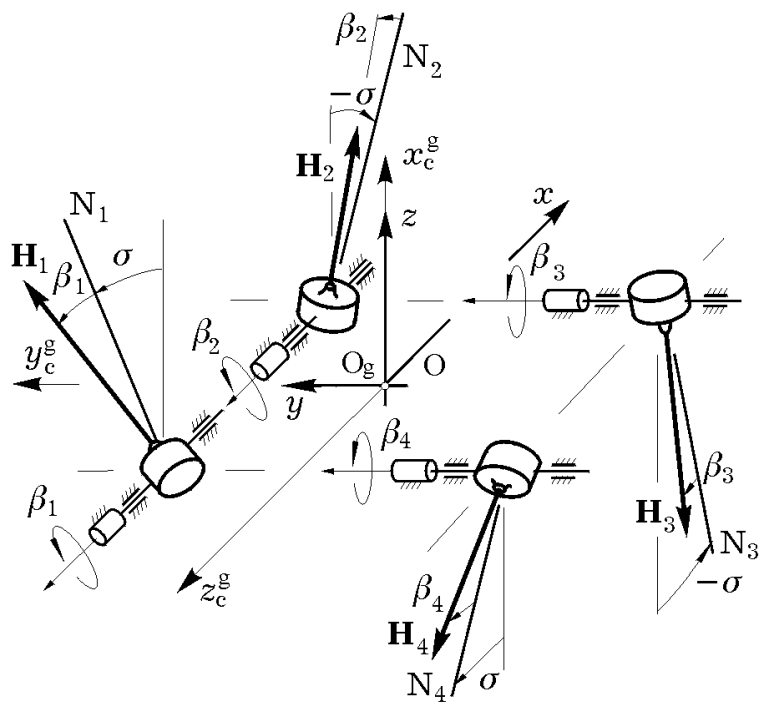

$a$
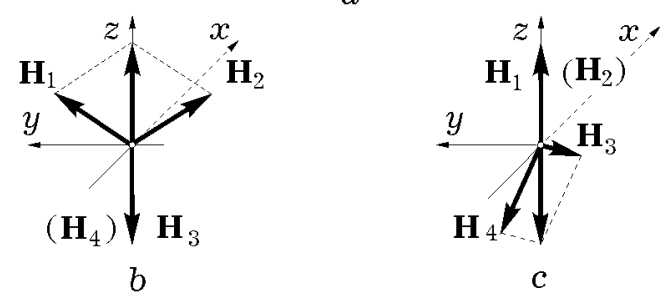

Fig. 1. The fault-tolerant "2-SPEED"-type minimal-redundant scheme of the MGC

\section{A MOMENT GYROCOMPLEX}

For information $\mathrm{SC}$ it is important to minimize the MGC mass and provide the possibility for reconfiguration of its structure and control algorithms for $2-3$ possible faults in any executive device of the MGC. The authors had been executed multilateral analysis of schemes for constructing the small-mass MGC based on RWs, various types of single- and two-gimbal GWs, the GDs with both the gear stepping drives (GSDs) and the moment gearless drives (MGDs) on their precession axes, in combination with unloading loops of accumulated angular momentum (AM) by reaction trusters and/or a magnetic torquers (Somov et al., 1999b).

For example, let the point $\mathrm{O}$ be the spacecraft mass center and $\mathrm{O} x y z$ is the body reference frame (BRF), see Fig. 1a. In the MGC canonical reference frame $\mathrm{O}_{\mathrm{g}} x_{c}^{\mathrm{g}} y_{c}^{\mathrm{g}} z_{c}^{\mathrm{g}}$ the angular momentum projections of the first (GD-1 \& GD-2) and the second (GD-3 \& GD-4) pairs of gyrodines always are summed up along the axis $\mathrm{O}_{\mathrm{g}} x_{c}^{\mathrm{g}}$. The GD's neutral positions $N_{p}, p=1: 4$ are directed at the angles $\pm \sigma$ with respect to positive (for the 1st GD's pair) and to negative (for the 2nd GD's pair) directions of the axis $\mathrm{O}_{\mathrm{g}} x_{c}^{\mathrm{g}}$, see Fig. $1 a$. Under the MGC Z -arrangement on the spacecraft body, when the axis $\mathrm{O}_{\mathrm{g}} x_{c}^{\mathrm{g}}$ is the same as the axis $\mathrm{O} z$ of $\mathrm{BRF}$, the following 4 efficient (for 3-axis spacecraft attitude control) GD configurations are possible on the basis of only 3 active gyrodynes:

- the configurations Z-I, I=1:4 - the MGC without GD-I, represented at the nominal state in Fig. $1 b$ (configurations Z-4 or Z-3) and in Fig. 1c, ( configurations Z-2 or Z-1).

So, the "2-SPEED"-type (Crenshaw, 1973) gyrocomplex scheme in Fig. $1 a$ is fault-tolerant under diagnostics of a faulted GD and the gyrocomplex reconfiguration by some passages between configurations Z-I on specific logic conditions.

\section{MATHEMATICAL MODELS}

The BRF attitude with respect to the inertial reference frame (IRF) is defined by quaternion $\boldsymbol{\Lambda}=\left(\lambda_{0}, \boldsymbol{\lambda}\right), \boldsymbol{\lambda}=\left(\lambda_{1}, \lambda_{2}, \lambda_{3}\right)$. Assume that $\boldsymbol{\Lambda}^{p}(t)$ is a quaternion, and $\boldsymbol{\omega}^{p}(t)=\left\{\omega_{i}^{p}(t)\right\}$ and $\dot{\boldsymbol{\omega}}^{p}(t)$ are angular rate and acceleration vectors of the programmed SC body's motion in the IRF. The error quaternion is $\mathbf{E}=\left(e_{0}, \mathbf{e}\right)=\tilde{\mathbf{\Lambda}}^{p}(t) \circ \boldsymbol{\Lambda}$, the Euler's parameters vector is $\mathcal{E}=\left\{e_{0}, \mathbf{e}\right\}$, the attitude error matrix is $\mathbf{C}_{e} \equiv \mathbf{C}(\mathcal{E})=\mathbf{I}_{3}-2[\mathbf{e} \times] \mathbf{Q}_{e}$, where $\mathbf{Q}_{e} \equiv \mathbf{Q}(\mathcal{E})=\mathbf{I}_{3} e_{0}+[\mathbf{e} \times]$ with $\operatorname{det}\left(\mathbf{Q}_{e}\right)=e_{0}$. Here symbols $\langle\cdot, \cdot\rangle, \times,\{\cdot\},[\cdot]$ for vectors and $[\mathbf{a} \times],(\cdot)^{\mathrm{t}}$ for matrix are conventional denotations.

The BRF attitude with respect to the orbital reference frame (ORF) is defined by quaternion $\boldsymbol{\Lambda}^{o}=\tilde{\boldsymbol{\Lambda}}_{o}(t) \circ \boldsymbol{\Lambda}$, where $\boldsymbol{\Lambda}_{o}$ is known quaternion of the ORF attitude with respect to the IRF, 
and also by vector of Euler's parameters $\mathcal{E}^{o}$, moreover the matrix $\mathbf{C}_{e}^{o}=\mathbf{C}\left(\mathcal{E}^{o}\right)$. For a fixed position of flexible structures on the $\mathrm{SC}$ body with some simplifying assumptions and for $t \in \mathrm{T}_{t_{0}}=$ $\left[t_{0},+\infty\right)$ a SC angular motion model appears as:

$$
\begin{aligned}
& \dot{\boldsymbol{\Lambda}}=\mathbf{\Lambda} \circ \boldsymbol{\omega} / 2 ; \mathbf{A}^{o}\{\dot{\boldsymbol{\omega}}, \ddot{\mathbf{q}}, \ddot{\boldsymbol{\beta}}, \dot{\boldsymbol{\Omega}}\}=\left\{\mathbf{F}^{\omega}, \mathbf{F}^{q}, \mathbf{F}^{\beta}, \mathbf{F}^{h}\right\},(11) \\
& \mathbf{F}^{\omega}=\mathbf{M}^{\mathrm{g}}-\boldsymbol{\omega} \times \mathbf{G}+\mathbf{M}_{d}^{o}+\mathbf{Q}^{o} ; \mathbf{M}^{\mathbf{g}}=-\dot{\mathcal{H}}=-\mathbf{A}_{\mathrm{h}} \dot{\boldsymbol{\beta}} ; \\
& \mathbf{F}^{q}=\left\{-a_{j j}^{q}\left(\left(\delta^{q} / \pi\right) \Omega_{j}^{q} \dot{q}_{j}+\left(\Omega_{j}^{q}\right)^{2} q_{j}\right)+\mathrm{Q}_{j}^{q}\left(\boldsymbol{\omega}, \dot{q}_{j}, q_{j}\right)\right\} ; \\
& \mathbf{F}^{\beta}=\mathbf{A}_{\mathrm{h}} \boldsymbol{\omega}+\mathbf{M}_{c}^{g}+\mathbf{M}_{d}^{g}+\mathbf{M}_{b}^{g}+\mathbf{M}_{f}^{g}+\mathbf{Q}^{g}(\cdot) ; \\
& \mathbf{F}^{h}=\mathbf{M}_{c}^{h}+\mathbf{M}_{d}^{h}+\mathbf{M}_{f}^{h}+\mathbf{Q}^{h}(\cdot) ; \mathbf{M}_{c}^{h}=\mathbf{M}^{h}+\mathbf{M}^{h a} ; \\
& \mathbf{A}^{o}=\left[\begin{array}{cccc}
\mathbf{J}^{o} & \mathbf{D}_{q} & \mathbf{D}_{g} & \mathbf{D}_{h} \\
\mathbf{D}_{q}^{\mathrm{t}} & \mathbf{A}^{q} & \mathbf{0} & \mathbf{0} \\
\mathbf{D}_{g}^{\mathrm{t}} & \mathbf{0} & \mathbf{A}^{g} & \mathbf{0} \\
\mathbf{D}_{h}^{\mathrm{t}} & \mathbf{0} & \mathbf{0} & \mathbf{A}^{h}=\mathbf{M}^{g}+\mathbf{M}^{g d}+\mathbf{M}^{g a} ; \\
\mathbf{G}=\mathbf{G}^{o}+\mathbf{D}_{q} \dot{\mathbf{q}}+\mathbf{D}_{g} \dot{\boldsymbol{\beta}} ; \\
\mathbf{G}^{o}=\mathbf{J}^{o} \boldsymbol{\omega}+\mathcal{H}(\boldsymbol{\beta}) ; \\
\mathbf{A}_{\mathrm{h}}=[\partial \mathcal{H}(\boldsymbol{\beta}) / \partial \boldsymbol{\beta}] ;
\end{array}\right.
\end{aligned}
$$$$
\mathbf{H}=\left\{\mathrm{H}_{p}\right\} ; \boldsymbol{\Omega}=\left\{\Omega_{p}\right\} ; \boldsymbol{\beta}=\left\{\beta_{p}\right\} ; \boldsymbol{\omega}=\left\{\omega_{i}\right\} ;
$$$$
\mathbf{q}=\left\{q_{j}\right\} ; \mathbf{H}_{p}\left(\beta_{p}\right)=\mathrm{H}_{p} \mathbf{h}_{p} ; \boldsymbol{H}(\boldsymbol{\beta})=\sum \mathbf{H}_{p}\left(\beta_{p}\right),
$$

where torques $\mathbf{M}_{d}^{g}$ and $\mathbf{M}_{d}^{h}$ of a physical damping, and also the electro-magnetic damper (EMD) torques $\mathrm{M}_{d p}^{g}\left(k_{d}^{g}, \dot{\beta}_{p}\right)$ with gain $k_{d}^{g}$ are nonlinear continuous functions; vectors of the rolling friction torques in bearings on the gyro-rotor (GR) axes $\mathbf{M}_{f}^{h}$ and on GD's precession axes $\mathbf{M}_{f}^{g}$, and also in general case the torque's vector $\mathbf{M}_{b}^{g}$ describing the influence of limiting supports on GD's precession axes, are nonlinear discontinuous functions.

The components of the MGC control vectors $\mathbf{M}_{c}^{g}$ and $\mathbf{M}_{c}^{h}$ with regard for the possible faults in electric circuits of MGDs or GSDs as well as the EMDs on the GD precession axes, and also that of the electric drives on the GD's rotor axes and arresters (cages) are described by hybrid functions

$$
\mathrm{M}_{p}^{x}=\sum_{l=1}^{2} \gamma_{p}^{f x l}(\nu) \gamma_{p}^{r x l}(\mu) a_{p}^{x} i_{p}^{x l}
$$

where $x=g, g d, g a, h, h a$, coordinates $\gamma_{p}^{y x l}, y=f, r$ are logic variables $\gamma_{p}^{y x l} \in\{0,1\} ; \gamma_{p}^{y x 1} \wedge \gamma_{p}^{y x 2}=0$; $\gamma_{p}^{y x 1} \vee \gamma_{p}^{y x 2}=1, p=1: 4 ; i_{p}^{x l}$ are the control currents and currents at the GD electro-magnetic arresters in main $(l=1)$ and in reserve $(l=2)$ circuits.

The functions $\gamma_{p}^{f x l}(\nu)$ are outputs of an ALA $\mathcal{A}^{f}$ with memory used for representing fault occurrences and damage development depending on the automaton time $\nu \in \mathbb{N}_{0}$. Functions $\gamma_{k}^{r x l}(\mu)$ are outputs of a SLA $\mathcal{A}^{r}$, also with memory, for description of damage's or fault's block-keeping and the reconfiguration sequence depending on the automaton time $\mu \in \mathbb{N}_{0}$. The currents in GD's control circuits $i_{p}^{g l}(t)$ for $\gamma_{p}^{r g l}=1$ and $i_{p}^{h l}(t)$ for $\gamma_{p}^{r h l}=1$ are proportional to GD's digital control voltages $u_{p}^{x}(t)=\operatorname{Zh}\left[\operatorname{Sat}\left(\operatorname{Qntr}\left(u_{p k}^{x}, b_{u}^{x}\right), B_{u}^{x}\right), \mathrm{T}_{u}\right]$, where $u_{p k}^{x}, x=g, h$ are the outputs of NCLs on the GDs precession and GRs axes, and functions $\operatorname{Sat}(x, a)$ and $\operatorname{Qntr}(x, a)$ are general-usage ones, while the holder model with the period $\mathrm{T}_{u}$ is of the type: $y(t)=\operatorname{Zh}\left[x_{k}, \mathrm{~T}_{u}\right]=x_{k} \quad \forall t \in\left[t_{k}, t_{k+1}\right)$.

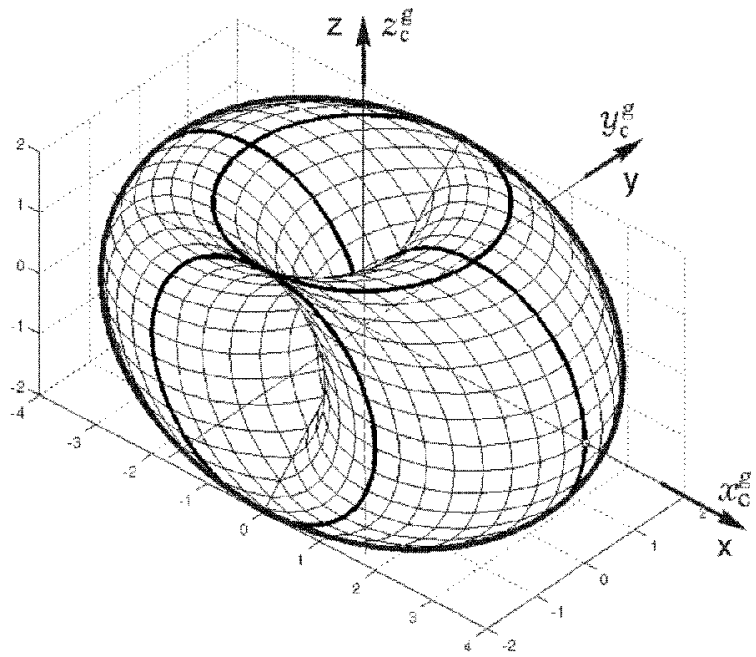

Fig. 2. Main singular sets of "2-SPEED" scheme

\section{THE MGC ADJUSTMENT}

Within precession theory of control moment gyros for $\mathrm{H}_{p}=h_{g}=$ const the MGC's AM vector is such:

$$
\begin{gathered}
\mathcal{H}(\boldsymbol{\beta})=h_{g} \mathbf{h}(\boldsymbol{\beta}) ; \mathbf{h}(\boldsymbol{\beta}) \equiv \sum \mathbf{h}_{p}\left(\beta_{p}\right)=\{\mathrm{x}, \mathrm{y}, \mathrm{z}\} \\
\mathrm{x}=\mathrm{x}_{12}+\mathrm{x}_{34} ; \mathrm{y}=\mathrm{y}_{1}+\mathrm{y}_{2} ; \mathrm{z}=-\left(\mathrm{z}_{3}+\mathrm{z}_{4}\right) ; \mathrm{x}_{p}=c_{\beta_{p}} ; \\
\mathrm{y}_{p}=s_{\beta_{p}} ; \quad \mathrm{z}_{p}=s_{\beta_{p}} ; \quad \mathrm{x}_{12}=\mathrm{x}_{1}+\mathrm{x}_{2} ; \quad \mathrm{x}_{34}=\mathrm{x}_{3}+\mathrm{x}_{4} .
\end{gathered}
$$
The MGC scheme have a lot of singular sets, main sets are presented in Fig. 2. The distribution law of normed $\operatorname{AM~} \mathbf{h}(\boldsymbol{\beta})$ between GD's pairs

$$
f_{\rho}(\boldsymbol{\beta})=\left(\tilde{\mathrm{x}}_{1}-\tilde{\mathrm{x}}_{2}\right)+\rho\left(\tilde{\mathrm{x}}_{1} \cdot \tilde{\mathrm{x}}_{2}-1\right)=0,
$$

with $\tilde{x}_{1}=x_{12} / q_{y} ; \tilde{x}_{2}=x_{34} / q_{z} ; q_{s}=\sqrt{4-s^{2}}$, $\mathrm{s}=\mathrm{y}, \mathrm{z}$, at "right-sided differential relay-hysteresis" tuning due to $D^{+} f_{\rho}(\boldsymbol{\beta})=\Phi_{\rho}\left(f_{\rho}(\boldsymbol{\beta}), \mathbf{h}(\boldsymbol{\beta})\right)$, see Somov (2001a), for the MGC's AM vector ensures its belonging to the imaginary singular sets only at separate time moments, and bijectively connects the vector $\mathbf{M}^{\mathbf{g}}$ with vectors $\boldsymbol{\beta}$ and $\dot{\boldsymbol{\beta}}$.

\section{SPACECRAFT CONTROL LAW}

If the error $\tilde{\boldsymbol{\omega}}$ in the angular rate vector $\boldsymbol{\omega}$ is defined as $\tilde{\boldsymbol{\omega}}=\boldsymbol{\omega}-\mathbf{C}_{e} \boldsymbol{\omega}^{p}(t)$, and the MGC's required control torque vector $\mathbf{M}^{\mathrm{g}}$ is formed as $\mathbf{M}^{\mathrm{g}}=\boldsymbol{\omega} \times \mathbf{G}^{o}+\mathbf{J}^{o}\left(\mathbf{C}_{e} \dot{\boldsymbol{\omega}}^{p}(t)-[\boldsymbol{\omega} \times] \mathbf{C}_{e} \boldsymbol{\omega}^{p}(t)+\tilde{\mathbf{m}}\right)$, then the simplest normed nonlinear model of the SC attitude error dynamics is as follows

$$
\dot{e}_{0}=-\langle\mathbf{e}, \tilde{\boldsymbol{\omega}}\rangle / 2 ; \quad \dot{\mathbf{e}}=\mathbf{Q}_{e} \tilde{\boldsymbol{\omega}} / 2 ; \quad \dot{\tilde{\boldsymbol{\omega}}}=\tilde{\mathbf{m}},
$$

for which the EFL synthesis results in the NCL

$$
\tilde{\mathbf{m}}(\mathcal{E}, \tilde{\boldsymbol{\omega}})=-\mathbf{A}_{0} \cdot \mathbf{e} \cdot \operatorname{Sgn}\left(e_{0}\right)-\mathbf{A}_{1} \cdot \tilde{\boldsymbol{\omega}},
$$

where $\mathbf{A}_{0}=\left(\left(2 a_{0}^{*}-\tilde{\omega}^{2} / 2\right) / e_{0}\right) \mathbf{I}_{3} ; \mathbf{A}_{1}=a_{1}^{*} \mathbf{I}_{3}-\mathbf{R}_{e \omega}$, $\operatorname{Sgn}\left(e_{0}\right)=\left(1\right.$, if $\left.e_{0} \geq 0\right) \vee\left(-1\right.$, if $\left.e_{0}<0\right)$, and matrix $\mathbf{R}_{e \omega}=\langle\mathbf{e}, \tilde{\boldsymbol{\omega}}\rangle \mathbf{Q}_{e}[\mathbf{e} \times] /\left(2 e_{0}\right)$. Moreover, the closedloop nonlinear system (13), (14) is transformed to a simplest linear system for the SC spatial attitude control. Simultaneously constructing the VLF's components using the Vandermonde matrix is analytically executed, the VLF's structure $\boldsymbol{v}(\mathbf{x})=$ $\boldsymbol{v}(\mathcal{E}, \tilde{\boldsymbol{\omega}})$ has become known, and for obtaining the inverse transformation $\mathbf{x}=\mathbf{\Psi}(\mathbf{z})$ in the explicit form it is sufficient to use the analytic inverse of matrix $\mathbf{Q}_{e}$ (Somov et al., 1999b). 
As result of the SC ACS (11) synthesis with the MGC scheme in Fig. 1a for the command order vector $\mathcal{E}_{\mathrm{c} k}^{o}$ and the measured vector $\mathcal{E}_{k}^{o f}$, the attitude error vector $\mathcal{E}_{k}^{\varepsilon}=\left\{\varepsilon_{0 k}, \boldsymbol{\varepsilon}_{k}\right\}$, matrix $\mathbf{A}_{\mathrm{h} k}^{\mathrm{f}}=\mathbf{A}_{\mathrm{h}}\left(\mathbf{H}_{k}^{\mathrm{f}}, \boldsymbol{\beta}_{k}^{\mathrm{f}}\right)$ and control vector $\mathbf{u}_{k}^{g}$ are computed by relations

$$
\begin{aligned}
& \varepsilon_{0 k}=e_{0 k}^{o f} e_{\mathrm{c} 0 k}^{o}+\left\langle\mathbf{e}_{k}^{o f}, \mathbf{e}_{\mathrm{c} k}^{o}\right\rangle ; \beta_{p k}^{\sigma \mathrm{f}}=\beta_{p k}^{\mathrm{f}}+(-1)^{p-1} \sigma ; \\
& \varepsilon_{k}=\mathbf{Q}\left(\mathcal{E}_{\mathrm{ck}}^{o}\right) \mathbf{e}_{k}^{o f}-e_{0 k}^{o f} \mathbf{e}_{\mathrm{c} k}^{o} ; \quad \boldsymbol{\epsilon}_{k}=-2 \varepsilon_{k} ; \\
& S_{h p k}^{\sigma \mathrm{f}}=\mathrm{H}_{p k}^{\mathrm{f}} \sin \beta_{p k}^{\sigma \mathrm{f}} ; \quad C_{h p k}^{\sigma \mathrm{f}}=\mathrm{H}_{p k}^{\mathrm{f}} \cos \beta_{p k}^{\sigma \mathrm{f}} \text {; } \\
& \mathbf{A}_{\mathrm{h} k}^{\mathrm{f}}=\left[\begin{array}{cccc}
-C_{h 3 k}^{\sigma \mathrm{f}} & -C_{h 4 k}^{\sigma \mathrm{f}} & 0 & 0 \\
0 & 0 & C_{h 1 k}^{\sigma \mathrm{f}} & C_{h 2 k}^{\sigma \mathrm{f}} \\
-S_{h 1 k}^{\sigma \mathrm{f}} & -S_{h 2}^{\sigma \mathrm{f}} & S_{h 3 k}^{\sigma \mathrm{f}} & S_{h 4 k}^{\sigma \mathrm{f}}
\end{array}\right] ; \\
& \mathbf{w}_{k}^{g}=\mathbf{w}_{k-1}^{g}+\mathrm{k}_{g}\left(\boldsymbol{\epsilon}_{k}+\mathrm{a}_{g} \boldsymbol{\epsilon}_{k-1}\right) ; \boldsymbol{\omega}_{o k}^{o f}=\mathbf{C}\left(\mathcal{E}_{k}^{o f}\right) \boldsymbol{\omega}_{o} ; \\
& \mathbf{u}_{k}^{g}=-\left(\mathbf{A}_{\mathrm{h} k}^{\mathrm{f}}\right)\left(\mathbf{w}_{k}^{g}+\boldsymbol{\omega}_{o k}^{o f}\right), k \in \mathbb{N}_{0},
\end{aligned}
$$

where $\mathrm{k}_{g}, \mathrm{a}_{g}$ are the constant scalar parameters.

Digital NCL (15) is universal for given MGC type, it provides the precise 3-axis attitude control of the SC body at the MGC reconfiguration, it is robust with respect to an accumulated $\mathrm{AM}$ of the "SC+MGC"-system and to variations of the SC inertial and rigidity characteristics. Operability of this NCL for large torque disturbances arising at the SC orbit correction as well as for spatial control of the SC body, flexible SAPs and RTAs, has been investigated.

\section{SOFTWARE AND SIMULATION}

The developed methods for dynamic research and design of robust fault-tolerant ACS had been implemented in the software system DYNAMICS and in well-known Matlab ${ }^{\mathrm{TM}}$. Some simulation results for the SC gyromoment ACS (11) see in Somov (2001a).

\section{CONCLUSIONS}

Contemporary nonlinear methods for design of robust fault-tolerant control systems applied at Russian information spacecraft (Kozlov et al., 1999; Batrenev et al., 1999), were presented. Designing such spacecraft ACSs have been conducted, including international projects: Ikar (Somov et al., 2001a) and Sesat (Somov et al., 2001b). Flight exploitation these ACSs have proved high efficiency of principles and methods employed for providing a robust fault-tolerant operation.

\section{REFERENCES}

Batrenev, V.A., V.A. Malyshev, V.A. Rayevsky, G.P. Titov and Ye.I. Somov (1999). Attitude and orbit control systems of Russian communication, geodetic and navigation spacecraft. Space Technology 19(3-4), 135-147.

Crenshaw, J.W. (1973). 2-SPEED, a single-gimbal control moment gyro attitude control systems. AIAA Paper (73-895), 1-10.
Frank, P.M., S.X. Ding and T. Marcu (2000). Model-based fault diagnosis in technical processes. Transactions of the Institute of Measurement and Control 22(1), 57-101.

Isidori, A. (1989). Nonlinear Control Systems. 2nd ed.. Springer-Verlag. New York.

Junkins, J.L. and J.D. Turner (1986). Optimal Spacecraft Rotational Maneuvers. Elsevier.

Kazantzis, N. and C. Kravaris (1997). Systemtheoretic properties of sampled-data representations of nonlinear systems obtained via Taylor-Lie series. International Journal of Control 67(6), 997-1020.

Kozlov, D.I., G.P. Anshakov, Yu.G. Antonov, V.P. Makarov and Ye.I. Somov (1999). Precision flight control of Russian remote sensing spacecraft. Space Technology 19(3-4), 149-163.

Kurakin, A.L. (1999). Computer Science: from Artificial Intelligent to Natural Tricks. Software \& Systems Intrnational (1), 2-5.

Schaub, H., S.R. Vadali and J.L. Junkins (1998). Feedback control law for variable speed control moment gyros. Journal of the Astronautical Sciences 46(3), 307-328.

Somov, Ye.I. (1997). Constructing the vector Lyapunov functions at synthesis of linear control systems with a partial measurement of state. Journal of Computer and Systems Sciences International 36(3), 73-86.

Somov, Ye.I. (2001a). Methods and software for research and design of the spacecraft robust fault tolerant control systems. Preprints of 15th IFAC Symposium on Automatic Control in Aerospace. Bologna. pp. 5-16.

Somov, Ye.I. (2001b). Robust stabilization of a flexible spacecraft at partial discrete measurement and a delay in forming control. Journal of Computer and Systems Sciences International 40(2), 287-307.

Somov, Ye.I., G.P. Anshakov and Yu.G. Antonov et al. (1999a). Ultra-precision attitude control of a large low-orbital space telescope. Control Engineering Practice 7(7), 1127-1142.

Somov, Ye.I., G.P. Anshakov and Yu.G. Antonov et al. $(2001 a)$. Dynamics of the maneuvering vehicle Ikar control system by the orbital placement of Globalstar satellites. Preprints of 15th IFAC Symposium on Automatic Control in Aerospace. Bologna. pp. 372-377.

Somov, Ye.I., V.A. Rayevsky and G.P. Titov (1999b). Fault-tolerant precision attitude control of communication and navigation spacecraft. Space Technology 19(3-4), 165-172.

Somov, Ye.I., V.A. Rayevsky and G.P. Titov et al. $(2001 b)$. Nonlinear dynamics of gyromoment attitude control system at communication satellite Sesat. Preprints of 5th IFAC Symposium "Nonlinear Control Systems". Vol. 5. IPME of RAS. St. Petersburg. pp. 1481-1486. 